\title{
Understanding student use of differentials in physics integration problems
}

\author{
Dehui Hu and N. Sanjay Rebello* \\ Department of Physics, 116 Cardwell Hall, Kansas State University, Manhattan, Kansas 66506-2601, USA
}

(Received 22 January 2013; published 26 July 2013)

\begin{abstract}
This study focuses on students' use of the mathematical concept of differentials in physics problem solving. For instance, in electrostatics, students need to set up an integral to find the electric field due to a charged bar, an activity that involves the application of mathematical differentials (e.g., $d r, d q$ ). In this paper we aim to explore students' reasoning about the differential concept in physics problems. We conducted group teaching or learning interviews with 13 engineering students enrolled in a secondsemester calculus-based physics course. We amalgamated two frameworks - the resources framework and the conceptual metaphor framework-to analyze students' reasoning about differential concept. Categorizing the mathematical resources involved in students' mathematical thinking in physics provides us deeper insights into how students use mathematics in physics. Identifying the conceptual metaphors in students' discourse illustrates the role of concrete experiential notions in students' construction of mathematical reasoning. These two frameworks serve different purposes, and we illustrate how they can be pieced together to provide a better understanding of students' mathematical thinking in physics.
\end{abstract}

DOI: 10.1103/PhysRevSTPER.9.020108

PACS numbers: 01.40.- $\mathrm{d}$

\section{INTRODUCTION}

Mathematical integration is widely used in many introductory and upper-division physics courses. Developing expertise to use integration in physics is both necessary and important for students to fully understand physics concepts as well as transfer their learning to new situations. Studies have reported that students are typically good at evaluating a predetermined mathematical integral, but often struggle with setting up integrals in physics problems $[1,2]$. Setting up integrals in a physics problem can be divided into several steps: setting up the expression for an infinitesimal quantity (e.g., $d E, d B$ ), accumulating the infinitesimal quantity, determining the variable of integration, and turning the integral into a form that can be evaluated mathematically [2]. Hence, using integration in physics requires students to not only understand the concept of an integral as representing a Riemann sum but also to correctly use a differential concept to express relations between physical quantities. For example, to construct the expression $d E=k d q / r^{2}$ from the fundamental Coulomb's law $E=k q / r^{2}$, a student needs to develop the understanding of the differential terms (i.e., $d q, d E$ ) in the context of an electric field. In this paper, we mainly focus on students' reasoning of the differential terms in physics equations as they solve physics integration problems.

Physicists construct notations based on a specific physical situation instead of following prescriptive

\footnotetext{
*srebello@phys.ksu.edu
}

Published by the American Physical Society under the terms of the Creative Commons Attribution 3.0 License. Further distribution of this work must maintain attribution to the author(s) and the published article's title, journal citation, and DOI. mathematical symbols [3]. For instance, mathematicians often use $x, y$ as variables and the integrals are often written in the form $\int f(x) d x$, whereas in physics the variables could be $r, l$, and $t$, each representing a different meaning associated with the corresponding physical system. The difference of using symbols in math and physics causes many difficulties for students as they use mathematical notations [4]. Differential terms are context dependent, often representing an infinitesimal amount or an infinitesimal change of a physical quantity. Compared with expert physicists, novice students have not developed a sophisticated understanding of mathematical concepts and they have less experience in physics problem solving. In this study, we investigate students' reasoning about differentials in physics problems requiring integration. We investigate student reasoning of this topic through the lens of resources [5] and conceptual metaphors [6].

There are three main purposes of this paper. First, we focus on the mathematical resources that students activate with their application of the differential concept in a physics context. The analysis of mathematical resources provides us with deeper insights into how students apply their mathematical learning to physics problem solving. Second, we identify the conceptual metaphors involved in students' use of mathematical differentials and prove the existence of metaphors in students' higher-level mathematical thinking (i.e., calculus). First studied in cognitive linguistics, conceptual metaphors are a kind of intuitive knowledge that describes mappings between the source domains, usually from our interactions with the physical world, and abstract concepts in target domains such as mathematics. Identifying the conceptual metaphors that appear in student discourse illustrates how concrete experiential notions might affect students' construction of scientific reasoning. 
It offers an alternative and perhaps fundamental explanation of student thinking. Third, we amalgamate the two frameworks-resources and conceptual metaphorswhich have previously typically been used separately. We illustrate how each framework serves different purposes and how they could be fused together to understand student reasoning.

In the next section, we give a brief review of related literature on students' understanding of a differential concept in the context of physics integration problems. In Sec. III, we discuss the conceptual framework that we chose for this study. In Sec. IV, we describe our research setting which includes the student population and methodology used to collect and analyze our data. In Sec. V, we present the results of our study. In Sec. VI, we discuss the possible implications for instruction. In Sec. VII, we discuss the main findings and the limitations of this study, as well as future work.

\section{RELEVANT LITERATURE}

In this section we review previous studies in two areas which are closely related to our work. First, we briefly discuss several prior studies about students' understanding of the differential concept in mathematics education. Since all of the students in our calculus-based physics classes have previously taken calculus, previous research in mathematics education provides us with the necessary background to understand their prior mathematics knowledge and what we could possibly observe in their mathematical thinking in physics contexts. It also allows us to see what mathematicians value in students' understanding of the differential concept and reflect on which of these attributes physicists might perceive as valuable in students' thinking. Next, we provide an overview of the literature on students' difficulties with setting up integrals from studies in physics education research. Our work is motivated and built upon these previous studies.

\section{A. Student understanding of calculus concepts}

Studies in mathematics education have shown that students are capable of following routine procedures to calculate a derivative, evaluate an integral, or find the area under a curve; however, they may lack the conceptual knowledge of those concepts [7,8]. Orton [8] conducted clinical interviews with 110 students to investigate their understanding of elementary calculus. Overall, he found that the errors made by students were structural (conceptual or fundamental) and students found that the execution of a procedure was relatively easy. For an interview on the topic of rate of change and differentiation, three main types of incorrect responses were apparent in students' understanding of the notion $d x$. Twenty-nine students explained $d x$ as "the differential of $x$ " or "the rate of change of $x$." An additional 25 students explained $d x$ as "the limit of $\delta x$ as $\delta x \rightarrow 0$." Another 20 students thought that $d x$ was an "amount of $x$ " or " $x$ increment"; in other words, it was more or less the same as $\delta x$. It is clear that students did not understand the symbols of differentiation or the approach to differentiation. Orton pointed out that "some students are introduced to differentiation as a rule to be applied without much attempt to reveal the reasons for and justifications of the procedure." In another interview about the integration concept, Orton found that very few students understood integration as the limit of a Riemann sum [1].

Artigue et al. [9] administered questionnaires to both physics and mathematics students to investigate their conceptions about differentials (e.g., $d x, d l$ ). In particular, she discussed two extreme tendencies in students' responses. First, the differential element lost all meaning except that it indicated the variable of integration which may have excluded other meanings. Second, the differential element had material content, such as " $d l$ is a small length" or "a little bit of wire." Artigue argued that the terms (e.g., small and little bit) were associated with "looseness in reasoning" indicating students' lack of a rigorous understanding of the mathematical concept, even though this reasoning is convenient and effective in physics.

Several other studies $[10,11]$ in math education also showed that students do not have a deep conceptual understanding of calculus concepts. Students learn the concepts as procedures and their understanding is often bereft of contextual meaning.

\section{B. Student application of calculus in physics}

In physics education research, several studies indicated students' lack of ability to apply the integral concept successfully in physics contexts $[2,12,13]$. In mathematics, students often learn how to follow an algorithm to evaluate an integral and operate symbols without referring to any concrete physical situations. Some of their conceptual difficulties with physics integration problems could be attributed to their insufficient understanding of mathematics concepts, such as their understanding of an integral as a sum and the area-integral relation [13-15]. In addition, a significant number of students have difficulties with setting up a physics problem rather than with the calculus aspect of the problem. In other words, their difficulty is related to the physics context, and not the procedural aspect of doing the calculus in the problem $[12,16]$.

When learning physics, students' mathematical reasoning could also be hindered by physical scenarios [3]. For instance, when teaching an integrated calculus and physics course, Yeatts and Hundhausen [3] found that most students could solve a problem presented in a calculus context; however, very few could solve the same problem correctly when it was presented in a physics context. Some studies investigated when and how students recognize the use of integration in physics $[12,17]$. Even though very few students did not realize an integral was required in a situation, most of them did not indicate an understanding 
of why the integration concept was applicable [17]. For instance, Cui et al. [12] found that when students were asked why integration was applicable to the problem they provided several responses, such as "the problems are similar to the examples before," "a quantity changes depends on another quantity," and "we need to sum up the small parts." Meredith and Marrongelle [17] described those different types of reasoning in terms of symbolic forms [18] as recall cue, dependence cue, and parts-of-awhole cue. The parts-of-a-whole cue is a more useful resource, but it is less commonly used by introductory students [2,17]. "How to decide the need of an integral" is the first important step for setting up integrals in physics contexts, but even students who activate more useful resources encounter several difficulties in setting up an integral [2].

Nguyen and Rebello [2] conducted individual teaching or learning interviews to investigate students' common difficulties when setting up integrals in electricity problems. They divided the process of applying integration to physics problems into four steps: recognizing the need for an integral, setting up the expression for the infinitesimal quantity, accumulating the infinitesimal quantity, and computing the integral. They pointed out that students' lack of understanding of the physical meaning in the infinitesimal term (e.g., $d x, d r$ ) caused them to ignore the infinitesimal term, to simply append it to the integrand, or even to prefix $d$ to whatever quantity was changing." When accumulating the infinitesimal quantity, almost all students started integrating the expression without attending to how these quantities should be added up. For instance, the electric field $d E$ is a vector quantity and the total electric field is the vector sum of $d E$. In the interview conducted by Nguyen and Rebello, eight out of 15 students did not realize they should integrate $d E$ by components. Students also encountered a number of difficulties in computing the integrals. Some could be attributed primarily to students' misunderstanding of the physical meaning of the symbols in the integrals. A few students still had difficulties in determining the limits of integrals.

Difficulties with setting up integrals are also prevalent with students in upper-division physics courses. Wilcox et al. [16] analyzed students' responses to traditional exam questions that involved finding the electric potential due to a continuous charge distribution in a junior level electricity and magnetism (E\&M) course. Students' common challenges included determining expressions for the differential charge element $(d q)$ and finding the limits of integration for a specific charge distribution. When setting up the integral, nearly half of the students had difficulty expressing the differential charge element.

Studies have reported students' difficulties when applying integration in physics; however, researchers have not carefully explored the students' reasoning underlying their difficulties. There is a gap between what mathematics education researchers have found about students' conceptual understanding in calculus and what physics education researchers have found about students' performance in physics problems. The main purpose of this study is to take one small step toward bridging this gap. Specifically, we focus on students' reasoning about differential concepts in the context of physics problems that require integration and how their reasoning influences students' setting up integrals in these problems. This paper builds on a recent conference proceedings paper [19] but involves much richer data, more comprehensive analysis, and many new findings.

\section{CONCEPTUAL FRAMEWORK AND RESEARCH QUESTIONS}

The main purpose of this study is to probe students' deep reasoning in applying mathematical concepts in physics contexts. We would like to situate ourselves in the knowledge-in-pieces tradition; in other words, we are interested in the richness and usefulness of students' thinking. Under the knowledge-in-pieces [20] tradition, we explore the various resources [5] that students use in their mathematical thinking in physics. In addition to the resources perspective, we use the conceptual metaphor theory to identify the fundamental coherent attributes underlying the resources from the language that students use as they reason about mathematics and physics concepts. Hence, we amalgamate aspects of two theoretical perspectives: the resources framework from physics education research and the conceptual metaphor theory from cognitive linguistics. In this section, we will review both perspectives and then provide a discussion of how we integrate the two perspectives in our research context.

\section{A. Resources perspective}

According to Hammer [5], resources are small-scale knowledge elements that we use in our everyday sense making. Generated from the knowledge-in-pieces [20] perspective, the resources model focuses on the role of prior knowledge that contributes to scientific learning. When learning physics, students use a variety of resources, such as their intuitive knowledge about the physical world $[21,22]$. A significant amount of research has investigated the role of intuitive knowledge played in formal physics learning [21-23]. When making sense of physical phenomena and processes, students use a form of intuitive knowledge through their interaction with the physical world, such as walking, pushing, pulling, and throwing objects. This intuitive sense of knowledge is often referred to as sense of mechanism or phenomenological primitives (p-prims) according to diSessa [21]. diSessa [21] identified several clusters of p-prims used by students when explaining the motion of objects in mechanics, such as Ohm's p-prims, force as mover, and dying away. This intuitive sense of knowledge could be applied directly to physics 
learning; however, if misapplied, it could cause conflicts and barriers for students' construction of formal physics knowledge.

Students can also activate a variety of resources from their mathematics knowledge, such as an intuitive sense of number, counting [24], and symbolic forms [18]. In order to understand how students interpret physics equations, Sherin [18] outlined the symbolic forms, i.e., cognitive mathematical primitives involved in student use of equations. A symbolic form consists of a symbol template and a conceptual schema. The conceptual schema is a simple structure associated with the symbolic form that offers a conceptualization of the knowledge contained in the mathematical expression [25]. As an example, the conceptual schema of balancing is associated with the mathematical template []$=[]$. In the physical situation "a book is at rest on a desk," the normal force acting on the book due to the desk is balanced by the gravitational force on the book due to Earth. It corresponds with the physics equation, $N_{\text {on } B}=W_{\text {on } B}$, which is a clear use of the symbol template []$=[]$ [25]. The symbolic forms draw the connections between the qualitative and quantitative reasoning of equations. In solving physics integration problems as discussed in the previous section, Meredith and Marrongelle [17] used the framework of symbolic forms to characterize the mathematical resources that cued students to use integration, including recall cue, dependence cue, and parts-of-awhole cue. Symbolic forms mainly describe algebraic equations; however, it is unclear whether this framework could be used in other types of equations, such as matrix equations or differential equations.

In addition to symbolic forms, several pieces of work examined students' use and development of mathematical resources in physics contexts requiring mathematics from different subfields [26,27]. Sayre and Wittmann [26] described students' creation of a coordinate system resource when choosing coordinate systems in physics mechanics problems. Black and Wittmann [27] identified a list of procedural mathematical resources from student work during the formation of the separate variables procedural resource in the context of solving differential equations in an intermediate mechanics problem. Unlike p-prims, these resources are less primitive, usually with internal structures, developed later in their life.

In this paper, we aim to find the mathematical resources involved in students' reasoning about differential terms in physics equations. By describing the kinds of mathematical resources activated by students, we will be able to better understand the mechanisms of student difficulties as described in our literature section.

\section{B. Conceptual metaphors}

Lakoff and Johnson introduced the theory of conceptual metaphor when studying the linguistic phenomenon of human beings [6]. This theory is based on the assumption that our ordinary conceptual system is metaphorically structured and metaphors are pervasive in our life, mostly reflected by the language we use. Linguistic evidence is an important source for exploring our intellectual system which governs how we think and behave. Conceptual metaphors describe the systematic mapping across multiple conceptual domains-concrete source domains and abstract target domains. The source domains are the mental schemata derived directly from sensorimotor experience, whereas target domains are domains formed by subjective or nonsensorimotor experience. According to Lakoff and Johnson [28], a conceptual metaphor allows the use of sensorimotor experiential-based schemata to conceptualize a subjective experience. In metaphorical cases such as "He is brought out of depression," experiencing the state of depression is associated with locations and the change of state is correlated with movement out of a location. "States are locations" is within the vast collections of primary metaphors identified by Lakoff and Johnson [28]. One important characteristic of a conceptual metaphor is that we typically conceptualize nonphysical notions like time, mind, emotion, and ideas in terms of concrete physical terms such as objects, substances, entities, and motions for which we have more experience.

Conceptual metaphors have also been used to characterize ideas in mathematical and scientific reasoning. Núñez brought the notion of embodiment cognition into mathematics and emphasizes the role our body and brain play in the construction of mathematical concepts [29]. He described conceptual metaphors as "fundamental cognitive mechanisms which project the inferential structure of a source domain onto a target domain, allowing the use of effortless species-specific body-based inference to structure abstract inference" [29]. Lakoff and Núñez described two main types of metaphorical mathematical ideas: grounding metaphors, which generate basic arithmetic notions (e.g., "addition as adding objects to a collection" within "arithmetic as an object collection"), and linking metaphors, which generate more sophisticated ideas connecting arithmetic to other branches of mathematics (e.g., "numbers as points on a line") [30]. Amin et al. [31] provided an analysis of the lay and scientific usage of the term energy from the conceptual metaphor perspective. In phrases involving constructing human activities, such as "full of energy" or "drained of energy," energy is understood as a possession contained in a person. In scientific use, energy transformation is often revealed in phrases such as "in some form" or energy "going back and forth between" different forms. The conceptualization of the energy concept is structured as a location event-structure metaphor, including submappings of forms of energy as locations and energy transformation as movements. The use of conceptual metaphors in scientific language has been described for a number of other topics, including thermodynamics [32] and quantum mechanics [33]. 
In our study, we use both resource and conceptual metaphor perspectives to analyze student reasoning. The two perspectives are closely interrelated but serve different purposes in the context of our research. First, metaphors are often regarded as kinds of intuitive resources used in everyday language and scientific reasoning. Second, conceptual metaphor theory also aligns well with the ontological categories of resources. Conceptual metaphors describe the mechanism of understanding abstract notions in terms of real objects. Chi and Slotta [34] described learners' ontologies when learning physics. They pointed out that students often think about physics concepts (e.g., light, electric current, and heat) as things (i.e., matter ontology); however, experts often classify those concepts as processes. More recently, Gupta, Hammer, and Redish [35] pointed out that this distinction is not necessarily universal, rather experts too, depending upon the context, use the matter ontology when describing phenomena. However, experts are more adept than novices at fluidly transitioning between ontologies. Hence, the conceptual metaphor perspective could perhaps offer us a more profound understanding of the resources that students use.

\section{Research questions}

In this study, we focus on students' use of mathematical resources associated with the differential concept as well as the conceptual metaphors that underlie their use of resources. Specifically, we address the following research questions:

(1) How do we characterize student use of resources about differentials in the context of physics problems requiring integration?

(2) What are the conceptual metaphors utilized by students along with their use of resources?

\section{METHODOLOGY}

In spring 2012, we conducted group teaching or learning interviews [36] with 13 students from a calculus-based physics course for engineers at a Midwestern university. This course is the second semester of a yearlong sequence of introductory physics courses for engineering majors. The instructional format is two regular lectures of 50 minutes each and two studio sessions of 110 minutes each. The lecture follows a traditional format with clicker questions and the studio is an integrated laboratory problem solving session [37].

We selected a sample of 13 student participants from a pool of 40 volunteers based on the convenience of schedules. One of the participants was a freshman and the rest were sophomores. Three participants were international students and two were female. All participants had taken prerequisite calculus of single variables (Calculus I and II). Although our problems only required the knowledge of single-variable calculus, eight of these participants had previously taken and two were concurrently taking calculus of multivariables (Calculus III). We organized students into five groups of two or three students each. In total, each group of students completed eight 75-minutelong interviews over the semester. For each interview, the interviewer met separately with each group. Each interview occurred within one week after students covered the related concepts in class. Problems presented to students were physics problems that required the use of integration in the contexts of electricity and magnetism. We gave students about three physics problems in each session and asked them to discuss the problems as a group using a whiteboard. Examples of problems are presented in Sec. V. During the interview sessions, the interviewer watched students work and asked them to elaborate on their thinking when necessary. If students did not know how to proceed, the interviewer provided them some hints so they could continue their work. In general, the interviewer did not point out students' mistakes or try to correct their thinking.

We videotaped and transcribed the interviews selectively for analysis. For this study, we focused our data analysis on students' reasoning about differentials. We used a phenomenographic approach to analyze the video transcripts, in that we did not decide a priori categories of students' responses, rather the categories were emergent from the data. The video transcripts were analyzed in several stages. First, we noted the various kinds of reasoning students provided about differentials as well as how often the same reasoning appeared in various settings. We selected the reasoning that appeared in more than two different settings. Next, we categorized each kind of reasoning as a resource and selected video clips with students' rich reasoning associated with each resource. We transcribed the video clips and conducted in-depth analysis based on information from student dialogues, gestures, and written work on the whiteboard. We identified the metaphorical phrases in students' conversations and analyzed the conceptual metaphors involved in metaphorical language. After generating the conceptual metaphors from the transcripts, we tried to match our metaphors with the metaphor categories described in the literature.

\section{RESULTS}

In this section we present our results in three subsections. First, we describe the mathematical resources that students activated with the use of the differential. Then we describe the conceptual metaphors involved in students' reasoning. Finally, we provide four case studies to illustrate students' activation of various mathematical resources about differential as well as conceptual metaphors in their mathematical reasoning. We also discuss how students activated the resources and used the conceptual metaphors in a physics context. 


\section{A. Description of mathematical resources}

We identified four resources about differentials that emerged from our interviews (Table I). We provide an overview of the four resources in this section. Then in Sec. VC we provide examples to demonstrate how students applied these resources in physics problems.

\section{Small amount}

Differential terms (e.g., $d x, d r, d E$ ) often contain concrete physical meanings based on physical systems. The fundamental idea of integration is the summation of infinitesimal quantities. When we apply the concept of an integral to physics, we often chop an object or a physical quantity into infinitesimal pieces and add the quantity or its effect due to each infinitesimal piece. Hence, we use differential terms to represent an infinitesimal piece or amount of a physical quantity. Some physicists may prefer to use less rigorous terms such as "extremely small or very small piece or segment" to describe the meaning of differential elements. Thus, we code this knowledge resource about differentials as small amount.

\section{Point}

In mathematics, differential terms such as $d x$ carry the meaning of an infinitesimal quantity. When constructing the expression for infinitesimal quantities, students perceive the differential term $d x$ as points on a line and they set up their equations accordingly. In Euclid's geometry, a point is defined as something that lacks all the dimensions or something with zero dimensions. In geometry, points often represent locations on a line, plane, or space. We discuss how students apply this resource in physics in our case studies.

\section{Differentiation}

One of the views about differentials held by students is related to the action of taking the derivative. In mathematics, $d$ is often used as a symbol for differentiation as in the equation $d f(x)=f^{\prime}(x) d x$, which involves differentiating a function with respect to a quantity. When solving physics

TABLE I. List of resources about differentials identified in our data set.

\begin{tabular}{ll}
\hline \hline Resources & Description \\
\hline Small amount & $\begin{array}{l}\text { A small amount of a physical quantity, e.g., a } \\
\text { very small length, a small amount of charge, } \\
\text { often aided by visual representations }\end{array}$ \\
Point & $\begin{array}{l}\text { A point with no dimensions, sometimes aided } \\
\text { by visual representations } \\
\text { Taking the derivative of a changing function, } \\
\text { often followed by mathematical operations }\end{array}$ \\
Differentiation & A variable that could be integrated \\
integration & \\
\hline \hline
\end{tabular}

integration problems, we find that some students interpret the symbol $d$ as a cue for a mathematical operation to take the derivative, which we refer to as the differentiation resource.

\section{Variable of integration}

For some students, the symbol $d$ indicates the variable of integration devoid of any physical meaning. In evaluating a mathematical integral, the notation $\int f(x)$ is commonly used by many students, simply leaving out the term $d x$ in the expression $\int f(x) d x$. When computing an integral, the only thing that students focus on is finding the antiderivative of $f(x)$. In physics problems, we often see that students do not include $d x$ or $d l$ as they set up equations and simply append it to the integrand in the end. Students do not see the differential term as representing any actual meaning other than an abstract, purely mathematical notion.

\section{B. Description of conceptual metaphors}

Throughout our interview sessions, there were a number of conceptual metaphors students activated while they set up integrals. To obtain a deeper understanding of the four resources we described above, we discuss several metaphors (Table II) that we identified from student work as they activated those resources in physics contexts.

\section{Objects metaphor}

This metaphor describes the mapping from a concrete object notion in their source domain based on physical experience to the abstract mathematical concept of differentials in the target domain. For example, students viewed the differential term $d x$ as a small segment of a bar and $d q$ as a small amount of charge. Students associated the differential terms with a small amount of a physical quantity in which the amount is an important attribute of an object. Thinking of differentials as objects helped students to quantify them in terms of the amount of length or charge, and to identify relations between different objects such as connecting the total length $L$ with a small length $d x$. The differentials as objects metaphor is an elaboration

TABLE II. List of conceptual metaphors associated with the use of differentials.

\begin{tabular}{ll}
\hline \hline $\begin{array}{l}\text { Conceptual } \\
\text { metaphors }\end{array}$ & Description \\
\hline Objects & $\begin{array}{l}\text { Differential terms represent an object, such as a } \\
\text { little charge or small length } \\
\text { Differential terms are locations in space, such } \\
\text { as points along a line }\end{array}$ \\
Machines & $\begin{array}{l}\text { Form } d[] \text { or } \int[] \text { is a machine that performs an } \\
\text { algorithm }\end{array}$ \\
Motion along & $\begin{array}{l}\text { In differential } d[], \text { the variable in the box is a } \\
\text { traveler moving along a line }\end{array}$ \\
\hline \hline
\end{tabular}


of the object event-structure metaphor cluster originally defined by Lakoff and Johnson [28]. The cluster of object event-structure metaphor involving the conceptualization of an abstract notion as a concrete object has been identified in everyday language, mathematical, and scientific reasoning.

Lakoff and Johnson [28] described two basic metaphors for understanding the internal structure of events and causes-the object and location event-structure metaphors. Event is a general term referring to anything that exists in space and time. The object and location event-structure metaphors are complex mappings with a number of submappings, though some may not be used. Consider the phrases "Harry has a headache" and "Harry gets a headache" in which headache is conceptualized as an object Harry possesses. This object event-structure metaphor includes submappings such as attributes are possessions and changes are movements in which the acquisition of an object ("gets a headache") is metaphorically described as the movement of an object (the object moves toward you). In mathematics, Lakoff and Núñez [30] elaborated on the cluster of object event-structure metaphor to describe metaphorical mathematical ideas in basic arithmetic notions, such as "addition as [in] adding objects to a collection." In thermodynamics, an application of the object event-structure metaphor is the entropy is considered as the possession of a system or a physical object in a container [31].

\section{Locations metaphor}

This metaphor involves the use of our everyday knowledge of motion in space, coming from our physical experience of movements, to understand the structure of events [28]. When interpreting the mathematical concept of differentials, in particular, spatial terms such as $d x, d A$ and $d V$, students conceptualize the differentials as locations in space. The differentials as locations metaphor aligns well with the basic location event-structure metaphor defined by Lakoff and Johnson [28]. In the example of location eventstructure metaphor involved in everyday language, "He is brought out of a depression," the state of depression is construed as location and the change of state is correlated with movement out of a location. In thermodynamics, the states of a system are understood as locations in cases like "the system is in equilibrium or at a temperature" [31].

\section{Machines metaphor}

This metaphor considers the understanding of the differential symbol $d[]$ as a machine that performs certain functions. Mathematicians also describe this type of metaphor as a grounding metaphor, meaning "ground our understanding of mathematical ideas in terms of everyday experience" [30]. Another example of a grounding metaphor is the mathematical notion of set is grounded in the notion of a container that comes from outside mathematics
[38]. Regarding the concept of function, one of the grounding metaphors is the function machine metaphor [30]. In the function machine metaphor, a function is regarded as a machine which performs an algorithm or calculates the input-output relationship. Similarly, the symbols $\int$ and $d$ can also be treated as operators that execute algorithms. When solving differentiation or integration problems, those symbols often appear in the structures of $d[]$ or $\int[]$, where the boxes contain variables or functions.

\section{Motion along a path metaphor}

In the motion along a path metaphor, the source domain depicts a fictitious motion along a trajectory. The target domain in our context involves the abstract notion of a differential concept. More specifically, when integrating over the differential terms $d[]$, the variable contained in the box is conceptualized as a traveler moving along a line. In the phrase "time flies" the passage of time is conceptualized as one moving along a path or observing a motion [28]. Mi-Kyung and Oh Nam [39] also identified students' use of a fictive motion metaphor when describing the solution of a differential equation. The solution is depicted as a trajectory of a moving point and the variables in the solution function are travelers moving along the axes.

\section{Case studies}

In this section we present four case studies to illustrate students' activation of mathematical resources and use of conceptual metaphors in their problem solving activities. As discussed in Sec. IV, our categorizations of mathematical resources and conceptual metaphors were those frequently used by students and they appeared in student reasoning in multiple contexts. These four case studies allow us to provide an in-depth discussion of student thinking. The four case studies involved two contextsfinding the total electric field due to a line of charge in episodes 1,2 , and 4 , as well as finding the total resistance of the cylindrical resistor with nonconstant resistivity in episode 3.

Setting up an integral to find the total electric field is one of the most common electrostatics problems and is familiar to students. Using the same physics scenario gives us the opportunity to see the variability of student reasoning and make comparisons between different groups of students. We do not have clear evidence to show that students explicitly use the differentiation resource in the context of electric field although students frequently use the words "the derivative of." Hence, we decided to choose an episode of student work in the context of resistance in which we have evidence to show that students activated the differentiation resource. We claim that students' activation of resources had strong contextual dependence; however, none of the resources were unique to a particular context. 


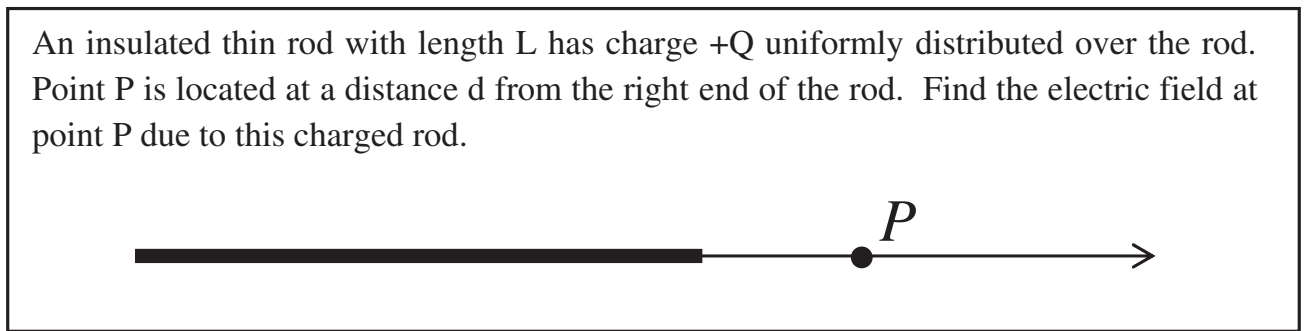

FIG. 1 (color online). Electric field problem.

\section{Episode 1: Student activation of the small amount resource}

This episode involves two students working on an electrostatic problem (Fig. 1), which we refer to as the line of charge problem. It was selected from the first interview session, which occurred in the second week of their course. This is a classic problem requiring integration in electrostatics. Before the interview students had attended the lecture on this topic in class and completed the related homework assignments. We select this episode from part of the total 20 minutes of conversation on this problem.

In order to find the total electric field at point $P$ due to this line of charge, the typical approach expert physicists take consists of three major steps. The first step is to chop the whole rod into infinitesimal pieces each of length $d x$, carrying an infinitesimal amount of charge $d q$. The second step is to set up the equation for $d E$ [Eq. (1)], which is the infinitesimal electric field at point $P$ due to $d q$ [Eq. (2)]. The last step is to find the total electric field $E$ by integrating $d E$ :

$$
\begin{aligned}
& d E=k \frac{d q}{r^{2}}, \\
& d q=\frac{Q}{L} d x .
\end{aligned}
$$

Two students (Dave and Alice) discuss how to set up the equation for $d q$.

Dave: I guess we can do separating little segments of $d q(s i c)$.

As they wrote down equations for $d E$ [Eq. (1)] and $d q$ [Eq. (2)], the interviewer prompted them to explain their thinking.

Dave: [looking at Eq. (1)] Well, since this is just the value that a particular line segment is putting on to our $P$, then that would only be a tiny segment of the charge, which is what we described as $d q$. And I believe $d q$ is what we have evaluated here [pointing to Eq. (2)]. So, if we plug in further, we can put that in.

Interviewer: Okay, can someone explain this equation? [pointing to Eq. (2)]

Alice: Well, we have a charge $Q$ over the entire length of $L$, so this is just saying when you have a little piece, cause you can write it differently, you can write it as $d q / d x=Q / L$. So then it is just a ratio of a whole charge over the whole length to a little bit of charge over a little bit of length.

Both Dave and Alice consistently used similar phrases such as "a tiny segment" or "a little bit" to describe the differential terms $d x$ and $d q$. When explaining Eq. (1), Dave used "a tiny segment of charge" to explain the meaning of $d q$ and "the value that a particular line segment is putting on to our $P$ " to describe $d E$. Apart from verbal explanations, he also used gestures to demonstrate a small section of the line by making a tiny interval between his thumb and index finger on the picture (Fig. 2). In describing her reasoning, Alice made use of contrastive terms, such as "the entire length" versus "a little piece" or "a whole charge" versus "a little bit of charge." Both students considered the symbol $d$ as representing a small piece or segment of a physical quantity in this context. Hence, we categorize this group of students as having applied the small amount resource when they set up the infinitesimal equation for $d E$ and $d q$.

Dave initiated the conversation by suggesting "we can do separating little segments of $d q$." By separating little segments he attempted to separate the whole rod into little segments of charge and sum up the electric field due to each segment of charge. We recognize that Dave activated the parts-of-a-whole resource. Sherin [18] described the parts of a whole as "amounts of generic substance associated with terms that contribute to a whole." Meredith and Marrongelle [17] found that students invoke the parts-of-awhole resource to cue the need of integration in physics when students interpret the integral as a sum of many contributions. When solving the line of charge problem, students first activated the parts-of-a-whole resource as a cue for integration and then used a small amount resource to set up expressions for infinitesimal quantities.

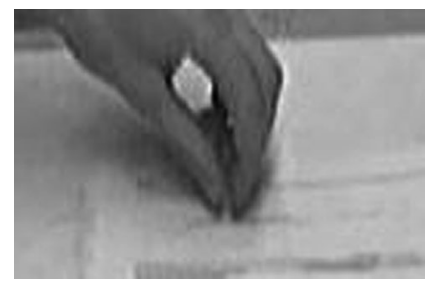

FIG. 2 (color online). Gestures in episode one. 
From the perspective of conceptual metaphors, students construct the abstract notion of differentials in terms of concrete objects such as the amount of a quantity. In their conversation, students used a number of terms such as "little segment" and "a little bit of..." to describe the differential terms $d x$ and $d q$. Near the end of the episode, in her response Alice says, "When you have a little piece." In this response, "a little piece" refers to a thing or object. This indicates that students viewed the mathematical notions $d x$ and $d q$ as substances or objects, an application of the object metaphor.

Students in this episode broke down the line into small line segments and then found the charge carried by each line segment. By viewing the total length $L$ as composed of very small line segments $d x$, students were able to construct ratios of the differential or infinitesimal terms (i.e., $d q, d x$ ) to finite quantities (i.e., $Q$ and $L$ ). Then students went on to build the expression for the infinitesimal charge element $d q$. This group eventually found the total electric field by adding up the electric field due to each charge element $d q$ successfully. The fundamental idea of integration is chopping the object into small pieces and adding the quantity (or effect) of each piece. Hence, we recognized the approach students invented to setting up an integral as chopping-adding pieces. The pieces correspond to the geometrical and physical constraints of the object. The geometrical constraint refers to the fact that the chopped pieces have the same dimension as the object, such as a line chopped into small line segments or an area chopped into small sections. The physical constraint determines how the object should be chopped. The use of the small amount resource and object metaphor helped students construct the abstract notions of differentials in terms of concrete things with which they have direct experience. In other words, it led students to make sense of the mathematics in physical scenarios.

\section{Episode 2: Student activation of point resource}

In the example of the line of charge problem (Fig. 1), two students (Aaron and Kelly) seemed to visualize the line as numbers of points each represented by $d x$. Near the beginning of solving this problem, they started to find the expression for infinitesimal charge element $d q$.

Aaron: Well, we are gonna find, like, would be like summing up little charges at every point?

Kelly: Yeah, so it's $d q, Q / L$ ?

Then, Kelly wrote down an equation for $d q$ [Eq. (3)] on the whiteboard.

$$
d q=\frac{Q}{L}
$$

Later on, before she proceeded to construct the integral equation for total electric field, she attempted to add $d x$ to the right-hand side of the equation, but she was not sure whether $d x$ should be included in the equation. Then the interviewer prompted the students to explain their thinking.

Interviewer: Can you guys explain this equation? What is the meaning of this equation [Eq. (3)]?

Aaron: The charge at every single point is charge divided by the distance.

Kelly: Basically, the point charge is at each point along $L$, is the total charge over its length, so like, what's it called?

Aaron: Charge density.

Later, the interviewer prompted

Interviewer: So what does $d q$ mean exactly?

Aaron: We knew we have to use integral to sum everything up. We need to know what we are summing up the whole time. So, we have to find...

Kelly: Just find the little charges by taking the total charge over the length it's over, to find... since it's uniform, we can find the charge at every point.

At the beginning, Aaron started to talk about "summing up little charges" which indicates that he realized that the total electric field is the sum of (the effect due to) little charges. Hence, Aaron seemed to have activated the partsof-a-whole resource. After that, they set up an equation for $d q$ which they described as "little charges" initially. Of particular interest in this episode is that there are two kinds of phrases emerging in their responses: charge at each point and point charge. Students used both phrases interchangeably in their conversation when describing the meaning of $d q$, and they did not appear to be aware that there are two ideas involved in the two phrases. From an expert's point of view, charge at each point involves a certain amount of charge located at each position on a line; whereas point charge involves viewing a certain amount of charge as a point quantity with no physical size. In other words, as students used the phrase "charge at each point," they implicitly associated $d q$ with a certain amount of charge and $d x$ with the location in which the charge $d q$ is located.

We also noticed that students used the phrases "little charge" in the beginning and the end of this episode and they used "point charge" elsewhere. It seems that students related both terms to the differential term $d q$, implying that they thought $d q$ represented a small amount of charge with negligible physical size. In physics when the size of an object can be neglected compared to the distance from the point of reference, the object is often viewed as a point for simplicity. For instance, in Newtonian mechanics we often use a point to represent the object and draw forces acting on the point, which we describe in a free-body diagram. The point model is also extensively used in E\&M, such as Coulomb's law, which defines electric force between two point charges. Thus, we conclude that students used the point resource in two different ways: differential term $d x$ represents a geometrical location in space and $d q$ represents a small amount of charge with negligible physical dimensions. 
In another context of finding the total charge of a disk with nonuniform charge distribution, we also found that students used similar reasoning (i.e., charge at each point on a disk) as they described $d A$ (i.e., small area element) and $d q$. In both geometrical shapes, students related the spatial differential terms $d x$ or $d A$ with a location in a line or plane. From the perspective of conceptual metaphors, students used the location metaphor in their reasoning about spatial differential terms such as $d x, d A$, and the object metaphor in their reasoning about $d q$ (i.e., amount of charge at each location).

In this episode, students invented a different approach towards setting up an integral, which we refer to as the separating-adding points approach. They considered the physical object as being separated into a number of points located on the object or small parts of the object. This approach differs from the chopping-adding pieces approach that we identified in the first episode. For example, a line might be viewed as a number of points located along the line rather than line segments; a surface area is viewed as points located on the surface rather than many small pieces of areas. As discussed above, when viewing the object as composed of points, students often failed to relate the points with physical dimensions. Students started with the idea of summing up little charges at every point. When talking about charges at each point students seemed to relate charge at a point to the ratio of total charge to the length. If there were discrete charges distributed and each point carried the same amount of charge, the charge at each point would be the total charge divided by the number of points. However, there was no discrete charge distribution. When constructing the equation relating the point charge with the ratio [Eq. (3)], students must have considered the length of the rod as a proxy for the number of points on the rod. Similarly, in another problem involving a charge disk with nonuniform charge distribution, we found that students set up an incorrect equation of $d q=Q / A$ for the differential charge element $d q$. We suspect that students linked the area $A$ of the disk with the numbers of points on the surface without noticing the difference between the two.
We infer that when thinking of the differential terms $d x$ or $d A$ as locations on a line or plane, students did not see the connection between the differential terms and amount of physical quantity as locations that are often regarded as having zero dimensions. Hence, the expression for infinitesimal quantities (e.g., $d q$ ) often did not include a spatial differential term (e.g., $d x, d A$ ).

\section{Episode 3: Student activation of a differentiation resource}

In the example of a resistor problem (Fig. 3), the resistivity is changing along the central axis of the cylindrical resistance; thus, integration is required to find the total resistance.

The basic resistance equation for a resistor with constant resistivity is given by Eq. (4). To find the infinitesimal expression for $d R$, two students (Zad and Alan) first made up a resistance function [Eq. (5)] by plugging the resistivity function into the basic resistance equation. They then found the expression for $d R$ [Eq. (6)] by taking the derivative of this function with respect to $x$ :

$$
\begin{gathered}
R=\frac{\rho L}{A}, \\
R=\frac{\rho(x) L}{A}, \\
d R=d\left(\frac{\rho(x) L}{A}\right)=\frac{d \rho(x)}{A} L .
\end{gathered}
$$

Interviewer: So why did you take the derivative of this? [pointing to Eq. (4)]

Zad: Um, because we just plugged it into... we plugged what $R$ was... into the integral of $R$ basically. So we need to take the derivative of it. So, we can plug into $R$. Because we basically pull $d x$ out of nowhere, because the derivative of the only changing function... then we require $d x$, we need to integrate that.

A material with length $\mathrm{L}$ and cross-sectional area A lies along the $x$-axis between $x=0$ and $x=\mathrm{L}$. Its resistivity varies along the rod according to $\rho(\mathrm{x})=\rho_{0} \cdot \mathrm{e}^{-\mathrm{x} / \mathrm{L}}$. Find the total resistance of this cylinder between two end faces.

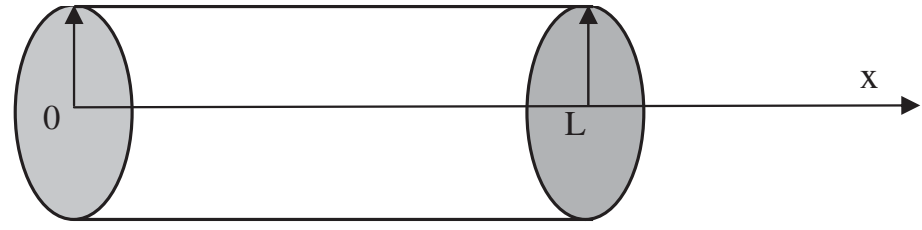

FIG. 3 (color online). Resistor problem. 
$\mathrm{Zad}$ : We have the function. You have to take the derivative so you can take the integral. I do not know how to explain it other than mathematically.

Alan: You need to take the integral across the whole thing. So, in order to do that, we have to do a derivative, but it's basically just taking the difference from one to the other.

In Zad's response, "changing function" refers to the resistivity of the cylinder as a function of $x$. In an earlier conversation, Zad said "the only variable was $x$, cause it's from 0 to $L$, so we have to take the integral of $x$ from 0 to $L$," which indicates that Zad used the function and variable as a cue for applying integration. Thus, we identified that Zad had activated the dependence symbolic form as described by Sherin [18] as "a whole depends on a particular symbol appears in the expression." When asked to explain how they set up the expression for $d R$ [Eq. (6)], both students provided similar reasoning - "you have to take the derivative so that you can take the integral." Students perceived $d R$ as taking the derivative of the resistance function, and that explains why they made up the resistance function [Eq. (5)]. Once they obtained the resistance function, they took the derivative of the resistance function as they had planned earlier. We recognize that this group of students activated the resource of differentiation when setting up the expression for infinitesimal resistance.

In their phrases such as "plug into the integral," "take the derivative," "take the integral," or "do the derivative," we claim that students treated the templates $d[]$ and $\int[]$ as machines; in other words, they used the machine metaphor. In order to find the output (i.e., total resistance), students figured out that the input of the machine $\int[]$ should be $d R$. Next, students needed to find a function as the input of the machine $d[]$. When no such function was available, they made up a resistance function [Eq. (5)] by plugging the resistivity function into the fundamental resistance equation.

Upon setting up an integral, the students' solution involved the purely mathematical process of finding a function, taking the derivative of the function, and taking the integral of the differential term. We refer to this chain of mathematical steps as the differentiating-integrating function approach. Another interesting phenomenon in the students' solution is that they never attempted to talk about the physical meaning of the equations or make use of visual representations (e.g., pictures). While applying this approach, the differentiation resource and machine metaphor are often embedded in student reasoning about the differential concept. The differential resource implies thinking of the symbol $d$ as taking the derivative of a certain function. It is very likely that the students' abstract notion of $d$ as an action comes from their experience of performing mathematical calculations. The machine metaphor maps the differential form $d[]$ to a machine that performs an algorithm. We could also argue that the students' notion about $d[]$ is grounded from their physical experience of machines which perform certain functions. Previous studies $[31,39]$ also found that the machine metaphor appeared to be prevalent in students' mathematical reasoning.

\section{Episode 4: Student activation of a variable of integration resource}

In this example, two students ( $\mathrm{Zad}$ and Alan) were discussing how to solve the line of charge problem (Fig. 1). They first set up the expression for $d q$ [Eq. (3)], which was incorrect. Then they moved on to set up the following integral equation:

$$
E=\int_{0}^{L} k \frac{d q}{(d+L-x)^{2}}
$$

However, they were not sure whether the numerator should be $d q$ or $(Q / L) d x$, even though they are in fact equivalent in this situation. In the following conversation, they tried to seek opinions from each other.

Zad: It would be $d q$ or $(Q / L) d x$ ?

Alan: Well, we are just looking at a small part of the bar right now.

Zad: The total $Q / L \ldots$ you don't need to take the derivative or anything, would just give you a basic number since ... [looks at the picture again] uniformly distributed over the rod, so you do not need $d q$, since there is not more charge, so it would be $(Q / L) d x$.

Alan: Our $q$ is not changing throughout the length, "cause it's uniform. So we should not need to integrate $q$. I didn't think.

When deciding whether $d q$ or $(Q / L) d x$ should be used as the numerator, both students agreed that $d q$ should not be used inside the integral as "there is no more charge" or " $q$ is not changing." Students thought any quantity $x$ should be a variable in the differential term $d x$ and could be integrated. In other words, the differential represents the variable of integration. We also noticed that students activated more than one resource about differentials. Prior to this conversation, students used the point resource to set up the equation for $d q$. In the conversation above, students also said they should "take the derivative" which is an indication of using the differentiation resource.

When they were discussing whether $d q$ or $(Q / L) d x$ should be included inside the integral, Alan talked about the fact that "our $q$ is not changing throughout the length." He appeared to be relating $d q$ with the notion that the charge is changing along the axis. However, this notion contradicted the fact that the charge is uniformly distributed along the line. Thus, students eventually decided not to use $d q$. In the case of $d x$, because the value of $x$ was changing along the axis, students decided to use $(Q / L) d x$. 
During the interviews, we found that when students wrote down integrals many often did not include the differential term such as $\int 2 \pi r$. And later on, they simply appended a differential term $(d r)$ to the integrand as they computed the integral. Consider the example of finding the total charge of a disk with nonuniform charge distribution in which we noticed students set up an integral $\int \sigma(r) \pi r^{2} d r$. As we asked students to explain the term $d r$, they said "as you integrate, you would go from 0 to $R$." We suspect that students might have perceived the differential term $d r$ as a traveler who moves along the radius from the starting point 0 to ending point $R$. From the conceptual metaphor perspective, students applied the motion along a path metaphor towards the understanding of mathematical differential.

\section{IMPLICATIONS}

This study has several important implications for instruction. First, our categorization of resources and conceptual metaphors provides us with deeper insights into students' underlying reasoning process. Students' approaches to physics problems are often more visible to instructors, but the reasoning process underlying their approaches is often less visible. A detailed analysis of students' reasoning can help instructors make inferences about what students might be thinking based on the observed students' work. For instance, when students write down an equation such as $d A=2 \pi r$ for an infinitesimal ring, some instructors might simply make judgments that the students just forgot to add $d r$ on the right-hand side of the equation due to their carelessness. However, it is very likely that students did not attribute any physical meaning to the term $d r$, which is consistent with the resource view of the differential element-the variable of integration. When thinking of the differentials simply as an indicator of doing integration, students encounter several conceptual difficulties in an unfamiliar situation even though they could survive by simply remembering the equations in familiar situations.

Second, we found that some resources or conceptual metaphors seem to be productive for students when solving electromagnetism problems. We provided a discussion about how students' use of mathematical resources and conceptual metaphors might lead to their approaches to setting up integrals in physics. This discussion may suggest directions for future research into which resources or metaphors are more productive in a larger student population and broader physics topics. While activating the small amount resource and object metaphor, students' solution involved chopping an object into pieces and adding the quantity or effect due to each piece (i.e., chopping-adding pieces approach), which seems to involve more mathematical sense making. Students appeared to be able to translate back and forth between the math and physics concepts. It seems that conceptualizing the symbol $d[]$ in terms of a concrete object made the abstract mathematical notion more transferable to physical scenarios. The separating-adding points approach is also consistent with the basic notion of chopping and adding; however, the use of the location metaphor diverts students' attention from reasoning about the dimensions of the infinitesimal terms and eventually seems to cause students difficulties in setting up expressions for infinitesimal quantities. In the differentiating-integrating function approach, students rely on mathematical operations without much qualitative or quantitative reasoning involved. Our detailed analysis of student responses showed that they applied the differentiation resource and machine metaphor.

Finally, our results can potentially guide the development of instructional strategies to facilitate students' use of differentials in physics. For instance, if students use the point resource, they may be unable to relate the differential terms to their respective dimensions. To help students relate the point quantity with its dimension, it is necessary to help students make the connection between the point and the amount carried by the point. Hence, it might be useful to lead students through the process of breaking down a rod into pieces and then shrinking each piece into infinitesimal length. Another possible strategy is to suggest a different metaphor (e.g., an object metaphor) that is more productive in this situation.

\section{CONCLUSIONS}

In this paper we have examined the resources and conceptual metaphors that are involved in students' application of differentials in physics problems requiring integration. We identified four resources and four conceptual metaphors in association with the differential concept. Analyzing students' reasoning from both perspectives allows us to obtain deeper and broader insights into students' mathematical thinking in physics contexts. We also provided multiple case studies to illustrate how students applied resources and used conceptual metaphors in various contexts of solving physics integration problems.

Our study contributes to both mathematics and physics education research on student use of integration in physics problem solving. While most studies have focused on what students are unable to do when solving integration problems, our study investigated the underlying reasoning processes that students involve in solving physics problems that use integration. Orton [8] and Artigue et al. [9] investigated students' understanding about the differential concept in mathematics; however, our study investigated the various mathematical resources and conceptual metaphors that students bring to bear while applying the differential concept in solving physics problems. Our study also contributes to the literature by providing evidence about the existence of conceptual metaphors involved in students' 
mathematical thinking and quantitative problem solving in introductory physics.

The small amount resource refers to a small portion of a physical object. This resource is used across many physics contexts, such as finding the electric field due to a charge distribution. An object metaphor is often involved with the use of a small amount resource. This metaphor allows students to associate the complex mathematical notions with their experiential knowledge of physical objects. The point resource involves two distinct meanings: one represents locations in space and the other represents a point quantity with negligible physical size. However, students seemed to be unaware of this distinction. Our analysis of student work suggested that students' notion of points as locations in space is embedded in their reasoning of spatial differential terms such as $d x, d A$. Students' notion of points as point quantities is associated with differential terms such as $d q$. The point resource used in the spatial differential terms also aligns with the location metaphor, in which students' metaphorical construct of differential terms as spatial locations might be grounded in their interactions with the physical world.

The differentiation resource involves associating differentials with the mathematical action of taking the derivative of a function. We identified the machine metaphor from students' linguistic use while solving integration problems. A machine usually yields an output from a given input. In fact, mathematical operators are often treated as machines and students are able to get the output when the input is explicitly given to them. However, our analysis of students solving physics problems finds that students tend to encounter difficulties if they need to find the input (e.g., a function) in a physical scenario when using this metaphor. The variable of integration resource implies that the symbol $d$ is purely an abstract entity, which is often followed by a variable. We consider it likely that students activated this resource when they simply dropped or added a differential term in an integral equation. In the motion along a path metaphor, students view the use of differentials as a traveler going from one point to another when doing integration. Mathematically, the concept of a differential has dual aspects: it is treated as an object representing an infinitesimal quantity and it is used as a mathematical operator for differentiating a function with respect to a variable. This dual aspect of a differential concept is also reflected in student use of conceptual metaphors.

Among the four mathematical resources about differentials, we found that the small amount and variable of integration are the most common resources that students used in various introductory electromagnetism problems. We have evidence that the point resource was used by two groups of students in several situations, such as finding the total electric field due to a charged bar and finding the total charge on a disk with nonuniform charge density. The differentiation resource appeared mostly in the work by one group of students in several different problem contexts, though other students also mentioned "the derivative of" occasionally. During the interview sessions, we found that some students seemed to have a relative robust reasoning about differentials, and they tended to activate the same resources across many different situations. For example, Dave and Alice (students in episode 1) used the small amount resource in almost all problem contexts; Zad (student in episodes 3 and 4) used the differentiation and variable of integration resources in many different contexts. Some other students' use of resources was more flexible and context sensitive.

We do not claim that applying unproductive resources or conceptual metaphors does not necessarily lead to the incorrectness of setting up an integral. For instance, in order to set up the infinitesimal expression, students need to break down the object in a correct way that goes beyond merely the activation of the small amount resource and object metaphor. The differentiating-integrating functions approach is not necessarily always an unproductive approach. When activating the differentiation resource and machine metaphor, if students could generate a function in a correct manner, they would eventually set up the integral correctly.

Our findings are based on interviews with 13 engineering students and it is very likely that there are other resources or conceptual metaphors that were not identified in our study. On the other hand, our eight interview sessions covered most of the physics topics in E\&M (secondsemester calculus-based physics) that involve the use of integration, such as the electric field due to a line or disk of charge, Gauss's law, resistance, Ampère's law, and BiotSavart's law. This study could potentially lead to a further study to investigate students' reasoning with a larger student population and broader range of physics topics, such as mechanics and thermodynamics. The quantitative study will potentially explore more resources or conceptual metaphors that were not identified in this study. It could also provide data to support the robustness and generalizability of each type of reasoning. One possible way is to design a two-level multiple-choice survey, in which students, in addition to selecting one of the choices, provide reasoning to support their choice or suggest alternatives that were not presented as part of the multiple choices. Such a survey would enable us to probe the resources and conceptual metaphors used by a larger student population, including those that were not previously uncovered in our study.

\section{ACKNOWLEDGMENTS}

This work is supported in part by the National Science Foundation Grant No. 0816207. The authors wish to thank Dr. Eleanor Sayre for her advice and suggestions regarding the use of the conceptual metaphors framework in our analysis. 
[1] A. Orton, Students' understanding of integration, Educ. Stud. Math. 14, 1 (1983).

[2] D. Nguyen and N.S. Rebello, Students' difficulties with integration in electricity, Phys. Rev. ST Phys. Educ. Res. 7, 010113 (2011).

[3] F. R. Yeatts and J.R. Hundhausen, Calculus and physics: Challenges at the interface, Am. J. Phys. 60, 716 (1992).

[4] E. F. Redish, in Proceedings of the Conference on World View on Physics Education: Focusing on Change, Delhi, 2005 (to be published).

[5] D. Hammer, Student resources for learning introductory physics, Am. J. Phys. 68, S52 (2000).

[6] G. Lakoff and M. Johnson, Metaphors We Live By (The University of Chicago Press, Chicago, 1980).

[7] N. Mahir, Conceptual and procedural performance of undergraduate students in integration, Int. J. Math. Educ. Sci. Technol. 40, 201 (2009).

[8] A. Orton, Students' understanding of differentiation, Educ. Stud. Math. 14, 235 (1983).

[9] M. Artigue, J. Menigaux, and L. Viennot, Some aspects of students' conceptions and difficulties about differentials, Eur. J. Phys. 11, 262 (1990).

[10] A. Bennett, T. Moore, and X. Nguyen, in Proceedings of the American Society for Engineering Education Annual Conference and Exposition, Vancouver, 2011 (to be published).

[11] V. Sealey, in Proceedings of the 28th Annual Meeting of the North American Chapter of the International Group for the Psychology of Mathematics Education, Merida, Mexico, 2006, edited by S. Alatorre, J. L. Cortina, M. Saiz, and A. Mendez (to be published), Vol. 2, pp. 46-53.

[12] L. Cui, N. S. Rebello, P. R. Fletcher, and A. G. Bennett, in Proceedings of the Annual Meeting of the National Association for Research in Science Teaching, San Francisco, CA, 2006 (unpublished).

[13] C. S. Wallace and S. V. Chasteen, Upper-division students' difficulties with Ampère's law, Phys. Rev. ST Phys. Educ. Res. 6, 020115 (2010).

[14] E. B. Pollock, J. R. Thompson, and D. B. Mountcastle, Student understanding of the physics and mathematics of process variables in $P-V$ diagrams, AIP Conf. Proc. 951, 168 (2007).

[15] R. R. Bajracharya, T.M. Wemyss, and J. R. Thompson, Student interpretation of the signs of definite integrals using graphical representations, AIP Conf. Proc. 1413, 111 (2012).

[16] B.R. Wilcox, M.D. Caballero, R.E. Pepper, and S.J. Pollock, Upper-division student understanding of Coulomb's law: Difficulties with continuous charge distributions, AIP Conf. Proc. 1513, 418 (2013).

[17] D. C. Meredith and K. A. Marrongelle, How students use mathematical resources in an electrostatics context, Am. J. Phys. 76, 570 (2008).

[18] B. L. Sherin, How students understand physics education, Cognit. Instr. 19, 479 (2001).

[19] D. Hu and N.S. Rebello, Characterizing student use of differential resources in physics integration problems, AIP Conf. Proc. 1513, 186 (2013).
[20] A. A. diSessa and B. L. Sherin, What changes in conceptual change?, Int. J. Sci. Educ. 20, 1155 (1998).

[21] A. A. diSessa, Toward an epistemology of physics, Cognit. Instr. 10, 105 (1993).

[22] D. Hammer, More than misconceptions: Multiple perspectives on student knowledge and reasoning, and an appropriate role for education research, Am. J. Phys. 64, 1316 (1996).

[23] B. Sherin, Common sense clarified: The role of intuitive knowledge in physics problem solving, J. Res. Sci. Teach. 43, 535 (2006).

[24] K. C. Fuson, in Handbook of Research on Mathematics Teaching and Learning, edited by D. A. Grouws (Macmillan, New York, 1992), pp. 243-275.

[25] J. Tuminaro, A Cognitive Framework for Analyzing and Describing Introductory Students' Use and Understanding of Mathematics in Physics (University of Maryland, College Park, MD, 2004).

[26] E. Sayre and M.C. Wittmann, Plasticity of intermediate mechanics students' coordinate system choice, Phys. Rev. ST Phys. Educ. Res. 4, 020105 (2008).

[27] K. Black and M. C. Wittmann, Procedural resource creation in intermediate mechanics, AIP Conf. Proc. 1179, 97 (2009).

[28] G. Lakoff and M. Johnson, Philosophy in the Flesh: The Embodied Mind and Its Challenges to Western Thought (Basic Books, New York, 1999), pp. 45-59.

[29] R.E. Núñez, in Proceedings of the 24th International Conference on Psychology of Mathematics Education, Hiroshima, Japan, 2000 (unpublished), pp. 3-22.

[30] G. Lakoff and R.E. Núñez, Where Mathematics Comes From: How the Embodied Mind Brings Mathematics into Being (Basic Books, New York, 2000).

[31] T. G. Amin, F. Jeppsson, J. Haglund, and H. Strömdahl, Arrow of time: Metaphorical construals of entropy and the second law of thermodynamics, Sci. Educ. 96, 818 (2012).

[32] F. Jeppsson, J. Haglund, T. G. Amin, and H. Strömdahl, Exploring the use of conceptual metaphors in solving problems on entropy, J. Learn. Sci. 22, 70 (2013).

[33] D. T. Brookes and E. Etkina, Using conceptual metaphor and functional grammar to explore how language used in physics affects student learning, Phys. Rev. ST Phys. Educ. Res. 3, 010105 (2007).

[34] M. T. H. Chi and J. D. Slotta, The ontological coherence of intuitive physics, Cognit. Instr. 10, 249 (1993).

[35] A. Gupta, D. Hammer, and E.F. Redish, The case for dynamic models of learners' ontologies in physics, J. Learn. Sci. 19, 285 (2010).

[36] P. V. Engelhardt, E. G. Corpuz, D. J. Ozimek, and N. S. Rebello, The teaching experiment-What it is and what it isn't, AIP Conf. Proc. 720, 157 (2004).

[37] C. M. Sorensen, A. D. Churukian, S. Maleki, and D. A. Zollman, The New Studio format for instruction of introductory physics, Am. J. Phys. 74, 1077 (2006).

[38] L. Bazzini, From grounding metaphors to technological devices: A call for legitimacy in school mathematics, Educ. Stud. Math. 47, 259 (2001).

[39] J. Mi-Kyung and K. Oh Nam, Analysis of students' use of metaphor: The case for a RME-based differential equations course, J. Korea Soc. Math. Educ. D 8, 19 (2004). 